\title{
Survival Estimation, Prognostic Factors Evaluation, and Prognostic Prediction Nomogram Construction of Breast Cancer Patients with Bone Metastasis in the Department of Bone and Soft Tissue Tumor: A Single Center Experience of 8 Years in Tianjin, China
}

\author{
Yao Xu, ${ }^{1,2}$ Haixiao Wu, ${ }^{1,2}$ Guijun Xu, ${ }^{2,3}$ Zhuming Yin,, Xin Wang, ${ }^{1,4}$ \\ Vladimir P. Chekhonin, ${ }^{2,5}$ Karl Peltzer, ${ }^{2,6} \mathrm{Shu} \mathrm{Li}^{2}$ Huiyang Li, ${ }^{2}$ Jin Zhang, \\ Wenjuan $M a \mathbb{D}^{1,2}$ and Chao Zhang $\mathbb{D}^{1,2}$ \\ ${ }^{1}$ Tianjin Medical University Cancer Institute and Hospital, National Clinical Research Center for Cancer, \\ Key Laboratory of Cancer Prevention and Therapy, Tianjin's Clinical Research Center for Cancer, Tianjin, China \\ ${ }^{2}$ The Sino-Russian Joint Research Center for Bone Metastasis in Malignant Tumor, Tianjin, China \\ ${ }^{3}$ Department of Orthopedics, Tianjin Hospital, Tianjin University, Tianjin, China \\ ${ }^{4}$ Department of Health Management Center (Epidemiology and Biostatistics), First Affiliated Hospital, Army Medical University, \\ Chongqing, China \\ ${ }^{5}$ Department of Basic and Applied Neurobiology, Federal Medical Research Center for Psychiatry and Narcology, Moscow, Russia \\ ${ }^{6}$ Department of Psychology, University of the Free State, Turfloop, South Africa
}

Correspondence should be addressed to Wenjuan Ma; mawenjuan2008@163.com and Chao Zhang; drzhangchao@tmu.edu.cn

Received 20 October 2021; Accepted 23 November 2021; Published 31 January 2022

Academic Editor: Sharad Goyal

Copyright ( $\odot 2022$ Yao Xu et al. This is an open access article distributed under the Creative Commons Attribution License, which permits unrestricted use, distribution, and reproduction in any medium, provided the original work is properly cited.

Purpose. Bone metastasis in breast cancer remains globally concerned. Accurate survival estimation would be beneficial for clinical decision-making, especially for the patients with potential indications of surgery. Based on a retrospective cohort from China, the study aimed to construct a prognostic prediction nomogram for breast cancer patients with bone metastasis. Methods. Breast cancer patients with bone metastasis diagnosed between 2009 and 2017 in our department were retrospectively selected. The total cohort was divided into construction and validation cohorts (ratio 7:3). A nomogram was constructed to predict the probability of survival, and the performance of model was validated. Results. A total of 343 patients were enrolled with 243 and 100 patients in construction and validation cohorts, respectively. The median overall survival for the total cohort was 63.2 (95\% CI: 52.4-74.0) months. Elevated ALP (HR=1.71, 95\% CI: 1.16-2.51; $P=0.006)$, no surgery for breast cancer (HR $=2.19,95 \% \mathrm{CI}: 1.30-3.70$; $P=0.003$ ), synchronous bone metastasis ( $\mathrm{HR}=1.98,95 \% \mathrm{CI}: 1.22-3.22 ; P=0.006)$, and liver metastasis $(\mathrm{HR}=1.68,95 \% \mathrm{CI}$ : 1.20-2.37; $P=0.003)$ were independent prognostic factors for worse survival. The independent predictors and other five factors (including age at diagnosis, ER status, PR status, Her-2 status, and the performance of bisphosphonate) were incorporated to construct the nomogram. The C-index was 0.714 (95\% CI: 0.636-0.792) and 0.705 (95\% CI: 0.705) in the construction cohort and validation cohort, respectively. All the calibration curves were close to the 45 -degree line, which indicated satisfactory calibration. Conclusion. A retrospective study aiming at prognostic estimation of breast cancer patients with bone metastasis was designed. Four independent prognostic factors were identified and a prognostic nomogram was constructed with satisfactory discrimination and calibration. The model could be used in survival estimation and individualized treatment planning. 


\section{Introduction}

Breast cancer (BC) is the most common primary malignant tumor in female. In 2021, there will be 281,550 estimated new cases and 43, 600 estimated deaths in the United States [1]. Due to the developed early detection and comprehensive treatment strategies, the prognosis of $\mathrm{BC}$ has been improved in recent years [2,3]. Despite of the improved prognosis of patients at early stage, metastatic breast cancer (MBC) attributed to the main cause of death among $\mathrm{BC}$ patients and it was reported that the 5-year breast cancerspecific survival (BCSS) was dismal 26\% [4]. Compared with other MBC patients, the survival of patients with breast cancer bone metastasis (BCBM) was better. The median survival of patients with bone-only metastasis was up to 24-54 months [5,6]. Due to the high heterogeneity of $\mathrm{BC}$, identification of prognostic factors and prediction of survival time was the prerequisite for individual decisionmaking.

Except the Department of Breast Cancer, bone metastasis in breast cancer was a common disease in the Department of Bone and Soft Tissue Tumor. Such patients usually chose the Department of Bone and Soft Tissue Tumor for skeletal-related events (SREs) and/or motor dysfunction. Compared with other departments, the patients with synchronous bone metastasis (SBM) are more common. The patients usually require the surgical intervention to release the symptom. Prognostic estimation was one of the most important issues before surgery performance.

Several prognostic factors of BCBM patients have been investigated in previous studies. A retrospective study on 238 cases concluded that breast subtype was associated with overall survival (OS), bone disease-free survival, and survival with bone disease [7]. The 5-year survival rate was up to $40 \%$ for luminal A, luminal B, and basal patients, while it was $4 \%$ for triple negative breast cancer patients [7]. Another study based on Surveillance, Epidemiology, and End Results (SEER) dataset attested that breast subtype, age at diagnosis, race, tumor grade, and the presence of organ metastasis were independent prognostic factors of BCBM patients [8]. Sufficient literatures on prognostic factors were reported, which was fundamental for subsequent study on survival estimation [9-12].

Clinical prediction models, usually incorporating patients' demographic and clinical characteristics, were an efficient tool to evaluate the probability of disease development and survival outcome $[13,14]$. As one of the most widespread predictive models, nomogram was widely reported in several types of cancers [15-17]. However, most prognostic nomograms among MBC patients were constructed based on SEER database [18, 19]. The generalizability in Chinese population remained unclear. Thus, the present study aimed to construct a prognostic prediction nomogram based on the cohort from the Department of Bone and Soft Tissue Tumor, Tianjin Medical University Cancer Institute and Hospital, Tianjin, China. The nomogram can help oncologists estimate prognosis accurately and guide the individualize treatment for Chinese patients.

\section{Materials and Methods}

2.1. Data Source and Cohort Selection. The medical records of breast cancer bone metastasis (BCBM) patients in the Department of Bone and Soft Tissue Tumors, Tianjin Medical University Cancer Institute and Hospital, were retrospectively collected. All of patients were older than 18 and diagnosed between January 2009 and December 2017. The exclusion criteria were as follows: (1) patients diagnosed with secondary primary cancer or multiple primary cancers; (2) patients diagnosed with bilateral primary breast cancer; (3) male patients; (4) foreign patients; (5) patients without detailed medical records; (6) patients without follow-up status; (7) patients with metabolic bone disease; and (8) patients with severe osteoporosis. The flowchart of the patient selection is listed in Figure 1.

2.2. Demographic and Clinical Variables. Patients' demographic and clinical characteristics were included as follows: age at diagnosis (18-45 years, 46-55 years, or $>55$ years), marital status (married or unmarried), history of smoking (yes or no), alcohol consumption (yes or no), menstrual status (menstruation or menopause), history of abortion (no or yes), family history of cancer (yes or no), histological type (ductal carcinoma or others), tumor grade (Grade I-II or Grade III), tumor size $(<2 \mathrm{~cm}, 2-5 \mathrm{~cm}$, or $>5 \mathrm{~cm})$, lymph node metastasis (yes or no), ER status (positive or negative), PR status (positive or negative), Her-2 status (positive or negative), Ki-67 status (positive or negative), the performance of surgery (yes or no), chemotherapy (yes or no), radiotherapy (yes or no), endocrinotherapy (yes or no), and targeted therapy (yes or no). The chemotherapy regimens included AC, CAF, and TAC in the present study.

Laboratory data were investigated and grouped according to normal threshold value in our hospital, including hemoglobin (HGB: 115-150 g/L, <115 g/L, or >150 g/L), carbohydrate antigen 153 (CA153: $0-25 \mathrm{U} / \mathrm{ml}$ or $>25 \mathrm{U} / \mathrm{ml}$ ), carcinoembryonic antigen (CEA: $0-5 \mathrm{ng} / \mathrm{ml}$ or $>5 \mathrm{ng} / \mathrm{ml}$ ), alkaline phosphatase (ALP: $50-135 \mathrm{U} / \mathrm{L},<50 \mathrm{U} / \mathrm{L}$, or $>135 \mathrm{U} /$ $\mathrm{L})$, and serum calcium $(2.10-2.55 \mathrm{mmol} / \mathrm{L},<2.10 \mathrm{mmol} / \mathrm{L}$, or $>2.55 \mathrm{mmol} / \mathrm{L}$ ). ABO blood type was also investigated, being grouped into A type, $\mathrm{B}$ type, $\mathrm{AB}$ type, and $\mathrm{O}$ type.

The records on organ metastasis, skeletal-related events (SREs), and associated treatment were included: the radiotherapy for bone metastatic site (no or yes), pathological fracture (no or yes), spinal cord compression (no or yes), surgery for bone metastatic site (no or yes), performance of bisphosphonate therapy (no or yes), liver metastasis (no or yes), brain metastasis (no or yes), lung metastasis (no or yes), and other organs metastasis (no or yes). As in previous study, synchronous bone metastasis (SBM) was defined as $\mathrm{BM}$ diagnosis within 6 months after BC diagnosis, while metachronous bone metastasis (MBM) was defined as BM diagnosis more than 6 months after $\mathrm{BC}$ diagnosis [20, 21].

2.3. Statistical Analysis. All missing values in the current study were completed by multiple imputation methods. The total cohort was randomly divided into the construction and 


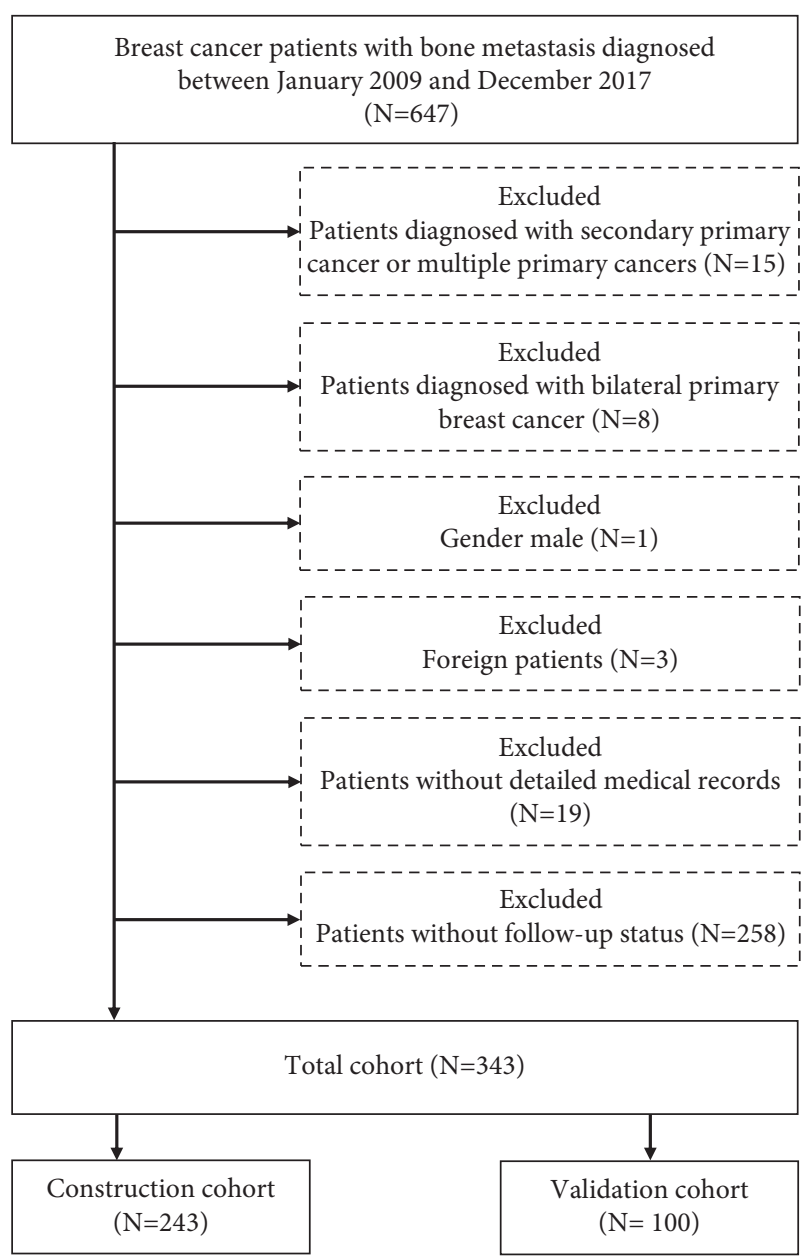

FIgUre 1: The flowchart of the patient selection.

validation cohort (ratio $7: 3$ ). The construction cohort was used to identify the prognostic factors for BCBM patients and to construct the predictive nomogram while the validation cohort was used to validate the performance.

Quantitative data were described as mean \pm standard deviation (SD) while categorical variables were presented as number and the percentage $(N, \%)$. The Cox proportional hazards regression was performed to identify the prognostic factors. The primary outcome was overall survival (OS), which was defined as the interval between the diagnosis of $\mathrm{BC}$ to all causes of death. The last follow-up date was on July 2020. The nomogram was formulated using the survival package in R. The discriminative ability of the model was evaluated with Harrell's concordance index (C-index) and receiver operating characteristic (ROC). The calibration curves (1000 bootstrap resamples) were performed to evaluate calibration ability of the nomogram.

The IBM SPSS Statistics (version 26.0, Armonk, NY, USA) was used for statistical analyses. The construction of prognostic nomogram and subsequent validation were performed with $\mathrm{R}$ version 4.0.0 (R Foundation for Statistical Computing, Vienna, Austria; https://www.r-project.org). All statistical tests were two-sided, and $P<0.05$ was considered significant.
2.4. Ethical Statement. The present study complied with the 1964 Helsinki Declaration and its later amendments or comparable ethical standards, and the Research Ethics Board of the Tianjin Medical University Cancer Institute and Hospital approved the study (bc2021010).

\section{Results}

3.1. Demographic and Clinicopathologic Characteristics. A total of 343 patients were eventually selected in the present study. After random grouping of the total cohort, there were 243 and 100 cases in the construction cohort and validation cohort, respectively.

For the total cohort, the median age was 48.0 (interquartile range, IQR: 41.0-57.0) years and patients with age $\leq 45$ years, $46-55$ years, and $>55$ years accounted for $39.1 \%, 31.8 \%$, and $29.2 \%$, respectively. The majority of patients were married $(N=324,94.5 \%)$. Most of the tumors were $2-5 \mathrm{~cm}(N=175,51.0 \%)$ and with positive nodal status $(N=276,80.5 \%)$. The tumor grade was I-II and III in $69.4 \%$ $(N=238)$ and $30.6 \%(N=105)$ of the patients. The percentages of patients with ER, PR, Her-2, and Ki-67 positive status were $69.1 \% \quad(N=237), \quad 56.6 \% \quad(N=194), \quad 21.6 \%$ $(N=74)$, and $25.4 \%(N=87)$, respectively. Distant metastasis was found among 119 patients in the liver, 36 patients in the brain, 137 patients in the lung, and 76 patients in other organs. More detailed information about demographic and clinicopathologic characteristics of the construction cohort and validation cohort is shown in Table 1 .

3.2. Synchronous Bone Metastasis and Metachronous Bone Metastasis. According to previous definition, there were 148 SBM patients and 195 MBM patients. The distribution of survival outcome of SBM and MBM patients is shown in Figure 2(a). For MBM patients, the mean interval between the diagnosis of breast cancer and bone metastasis was 61.9 (6.2-225.2) months. The cumulative frequency is shown in Figure 2(b).

For SBM patients, 95.9\% (142/148) presented axial skeleton (including the skull and vertebrae) metastasis while $46.6 \%(69 / 148)$ presented appendicular skeleton metastasis. The number of patients with axial skeleton and appendicular skeleton metastasis were $192(98.5 \%)$ and $98(50.3 \%)$ in MBM patients.

\subsection{Survival and Prognostic Factors of Breast Cancer Patients} with BM. At the last follow-up, a total of 273 patients deceased. The mean OS was 86.3 (95\% CI: 77.1-95.6) months and the median OS was 63.2 (95\% CI: 52.4-74.0) months. The 1-, 2-, 5-, and 10-year survival rates were $88.9 \%, 69.3 \%$, $52.8 \%$, and $25.8 \%$, respectively.

In the construction cohort, a total of 190 patients died at the last follow-up. The mean OS and the median OS were 92.8 (95\% CI: $80.7-104.9$ ) and 70.0 (95\% CI: 59.2-80.8) months, respectively. The 1-, 2-, 5-, and 10-year survival rates were $90.5 \%, 71.9 \%, 56.3 \%$, and $27.3 \%$, respectively. The following variables were associated with survival: age at diagnosis, ABO blood type, CA153, ALP, tumor grade, 
TABle 1: Demographic and clinicopathologic characteristics in the present study.

\begin{tabular}{|c|c|c|c|c|c|}
\hline Subject characteristics & $\begin{array}{c}\text { Total cohort } \\
N(\%)\end{array}$ & $\begin{array}{c}\text { Construction cohort } \\
N(\%)\end{array}$ & $\begin{array}{c}\text { Validation cohort } \\
N(\%)\end{array}$ & $x^{2}$ & $P$ value \\
\hline \multicolumn{6}{|l|}{ Age } \\
\hline $18-45$ & $134(39.1)$ & $98(40.3)$ & $36(36.0)$ & \multirow{3}{*}{0.594} & \multirow{3}{*}{0.743} \\
\hline $46-55$ & $109(31.8)$ & $75(30.9)$ & $34(34.0)$ & & \\
\hline$>55$ & $100(29.2)$ & $70(28.8)$ & $30(30.0)$ & & \\
\hline \multicolumn{6}{|l|}{ Marital status } \\
\hline Married & $324(94.5)$ & $228(93.8)$ & $96(96.0)$ & \multirow[b]{2}{*}{0.639} & \multirow[b]{2}{*}{0.424} \\
\hline Unmarried & $19(5.5)$ & $15(6.2)$ & $4(4.0)$ & & \\
\hline \multicolumn{6}{|l|}{ History of smoking } \\
\hline Yes & $17(5.0)$ & $11(4.5)$ & $6(6.0)$ & \multirow{2}{*}{0.326} & \multirow{2}{*}{0.568} \\
\hline No & $326(95.0)$ & $232(95.5)$ & $94(94.0)$ & & \\
\hline \multicolumn{6}{|l|}{ Alcohol consumption } \\
\hline Yes & $14(4.1)$ & $12(4.9)$ & $2(2.0)$ & \multirow{2}{*}{1.562} & \multirow{2}{*}{0.211} \\
\hline No & $329(95.9)$ & $231(95.1)$ & $98(98.0)$ & & \\
\hline \multicolumn{6}{|l|}{ Menstrual status } \\
\hline Menstruation & $108(31.5)$ & $80(32.9)$ & $28(28.0)$ & \multirow{2}{*}{0.796} & \multirow{2}{*}{0.372} \\
\hline Menopause & $235(68.5)$ & $163(67.1)$ & $72(72.0)$ & & \\
\hline \multicolumn{6}{|l|}{ History of abortion } \\
\hline No & $236(68.8)$ & $162(66.7)$ & $74(74.0)$ & \multirow{2}{*}{1.775} & \multirow{2}{*}{0.183} \\
\hline Yes & $107(31.2)$ & $81(33.3)$ & $26(26.0)$ & & \\
\hline Family history of tumor & & & & & \\
\hline Yes & $83(24.2)$ & $61(25.1)$ & $22(22.0)$ & & \\
\hline No & $260(75.8)$ & $182(74.9)$ & $78(78.0)$ & 0.372 & 0.542 \\
\hline ABO blood type & & & & & \\
\hline A type & $90(26.2)$ & $66(27.2)$ & $24(24.0)$ & & \\
\hline B type & $116(33.8)$ & $82(33.7)$ & $34(34.0)$ & & \\
\hline $\mathrm{AB}$ type & $46(13.4)$ & $31(12.8)$ & $15(15.0)$ & 0.549 & 0.908 \\
\hline O type & $91(26.5)$ & $64(26.3)$ & $27(27.0)$ & & \\
\hline$H G B$ & & & & & \\
\hline $115-150 \mathrm{~g} / \mathrm{L}$ & $276(80.5)$ & $194(79.8)$ & $82(82.0)$ & & \\
\hline$<115 \mathrm{~g} / \mathrm{L}$ & $59(17.2)$ & $43(17.7)$ & $16(16.0)$ & 0.227 & 0.893 \\
\hline$>150 \mathrm{~g} / \mathrm{L}$ & $8(2.3)$ & $6(2.5)$ & $2(2.0)$ & & \\
\hline CA153 & & & & & \\
\hline $0-25 \mathrm{U} / \mathrm{ml}$ & $113(32.9)$ & $75(30.9)$ & $38(38.0)$ & & \\
\hline$>25 \mathrm{U} / \mathrm{ml}$ & $230(67.1)$ & $168(69.1)$ & $62(62.0)$ & 1.633 & 0.201 \\
\hline$\overline{C E A}$ & & & & & \\
\hline $0-5 \mathrm{ng} / \mathrm{ml}$ & $171(49.9)$ & $116(47.7)$ & $55(55.0)$ & & \\
\hline$>5 \mathrm{ng} / \mathrm{ml}$ & $172(50.1)$ & $127(52.3)$ & $45(45.0)$ & 1.495 & 0.221 \\
\hline$A L P$ & & & & & \\
\hline $50-135 \mathrm{U} / \mathrm{L}$ & $227(66.2)$ & $164(67.5)$ & $63(63.0)$ & & \\
\hline$<50 \mathrm{U} / \mathrm{L}$ & $26(7.6)$ & $18(7.4)$ & $8(8.0)$ & 0.659 & 0.719 \\
\hline$>135 \mathrm{U} / \mathrm{L}$ & $90(26.2)$ & $61(25.1)$ & $29(29.0)$ & & \\
\hline Serum calcium & & & & & \\
\hline $2.10-2.55 \mathrm{mmol} / \mathrm{L}$ & $228(66.5)$ & $165(67.9)$ & $63(63.0)$ & & \\
\hline$<2.10 \mathrm{mmol} / \mathrm{L}$ & $38(11.1)$ & $27(11.1)$ & $11(11.0)$ & 1.050 & 0.592 \\
\hline$>2.55 \mathrm{mmol} / \mathrm{L}$ & $77(22.4)$ & $51(21.0)$ & $26(26.0)$ & & \\
\hline Pathology & & & & & \\
\hline Ductal carcinoma & $219(63.8)$ & $155(63.8)$ & $64(64.0)$ & & \\
\hline Others & $124(36.2)$ & $88(36.2)$ & $36(36.0)$ & 0.001 & 0.970 \\
\hline Grade & & & & & \\
\hline I-II & $238(69.4)$ & $172(70.8)$ & $66(66.0)$ & & \\
\hline III & $105(30.6)$ & $71(29.2)$ & $34(34.0)$ & 0.763 & 0.382 \\
\hline Tumor size & & & & & \\
\hline$<2 \mathrm{~cm}$ & $123(35.9)$ & $80(32.9)$ & $43(43.0)$ & & \\
\hline $2-5 \mathrm{~cm}$ & $175(51.0)$ & $129(53.1)$ & $46(46.0)$ & 3.187 & 0.203 \\
\hline$>5 \mathrm{~cm}$ & $45(13.1)$ & $34(14)$ & $11(11.0)$ & & \\
\hline
\end{tabular}


TABle 1: Continued.

\begin{tabular}{|c|c|c|c|c|c|}
\hline Subject characteristics & $\begin{array}{c}\text { Total cohort } \\
N(\%) \\
\end{array}$ & $\begin{array}{c}\text { Construction cohort } \\
N(\%) \\
\end{array}$ & $\begin{array}{c}\text { Validation cohort } \\
N(\%) \\
\end{array}$ & $x^{2}$ & $P$ value \\
\hline \multicolumn{6}{|l|}{ Lymph node metastasis } \\
\hline Yes & $276(80.5)$ & $198(81.5)$ & $78(78.0)$ & \multirow{2}{*}{0.546} & \multirow{2}{*}{0.460} \\
\hline No & $67(19.5)$ & $45(18.5)$ & $22(22.0)$ & & \\
\hline \multicolumn{6}{|l|}{ ER status } \\
\hline Positive & $237(69.1)$ & $165(67.9)$ & $72(72.0)$ & \multirow[b]{2}{*}{0.557} & \multirow{2}{*}{0.455} \\
\hline Negative & $106(30.9)$ & $78(32.1)$ & $28(28.0)$ & & \\
\hline \multicolumn{6}{|l|}{ PR status } \\
\hline Positive & $194(56.6)$ & $134(55.1)$ & $60(60.0)$ & \multirow{2}{*}{0.680} & \multirow{2}{*}{0.410} \\
\hline Negative & $149(43.4)$ & $109(44.9)$ & $40(40.0)$ & & \\
\hline \multicolumn{6}{|l|}{ Her-2 status } \\
\hline Positive & $74(21.6)$ & $51(21.0)$ & $23(23.0)$ & \multirow{2}{*}{0.170} & \multirow{2}{*}{0.681} \\
\hline Negative & $269(78.4)$ & $192(79.0)$ & $77(77.0)$ & & \\
\hline \multicolumn{6}{|l|}{ Ki-67 status } \\
\hline Positive & $87(25.4)$ & $60(24.7)$ & $27(27.0)$ & \multirow{2}{*}{0.199} & \multirow{2}{*}{0.655} \\
\hline Negative & $256(74.6)$ & $183(75.3)$ & $73(73.0)$ & & \\
\hline \multicolumn{6}{|l|}{ Surgery for $B C$} \\
\hline Yes & $262(76.4)$ & $186(76.5)$ & $76(76.0)$ & \multirow{2}{*}{0.012} & \multirow{2}{*}{0.914} \\
\hline No & $81(23.6)$ & $57(23.5)$ & $24(24.0)$ & & \\
\hline \multicolumn{6}{|l|}{ Chemotherapy } \\
\hline No & $21(6.1)$ & $11(4.5)$ & $10(10.0)$ & & \\
\hline Yes & $322(93.9)$ & $232(95.5)$ & $90(90.0)$ & 3.692 & 0.055 \\
\hline Radiotherapy & & & & & \\
\hline No & $225(65.6)$ & $160(65.8)$ & $65(65.0)$ & & \\
\hline Yes & $118(34.4)$ & $83(34.2)$ & $35(35.0)$ & 0.022 & 0.881 \\
\hline Endocrinotherapy & & & & & \\
\hline No & $183(53.4)$ & $129(53.1)$ & $54(54.0)$ & & \\
\hline Yes & $160(46.6)$ & $114(46.9)$ & $46(46.0)$ & 0.024 & 0.878 \\
\hline Targeted therapy & & & & & \\
\hline No & $302(88.0)$ & $212(87.2)$ & $90(90.0)$ & & \\
\hline Yes & $41(12.0)$ & $31(12.8)$ & $10(10.0)$ & 0.512 & 0.474 \\
\hline Time of $B M$ & & & & & \\
\hline SBM & $148(43.1)$ & $104(42.8)$ & $44(44.0)$ & & \\
\hline MBM & $195(56.9)$ & $139(57.2)$ & $56(56.0)$ & 0.042 & 0.838 \\
\hline Radiotherapy for BM & & & & & \\
\hline No & $283(82.5)$ & $202(83.1)$ & $81(81.0)$ & & \\
\hline Yes & $60(17.5)$ & $41(16.9)$ & $19(19.0)$ & 0.222 & 0.637 \\
\hline Pathological fracture & & & & & \\
\hline No & $310(90.4)$ & $222(91.4)$ & $88(88.0)$ & & \\
\hline Yes & $33(9.6)$ & $21(8.6)$ & $12(12.0)$ & 0.919 & 0.338 \\
\hline Spinal cord compressior & & & & & \\
\hline No & $333(97.1)$ & $236(97.1)$ & $97(97.0)$ & & \\
\hline Yes & $10(2.9)$ & $7(2.9)$ & $3(3.0)$ & 0.004 & 0.952 \\
\hline Surgery for $B M$ & & & & & \\
\hline No & $334(97.4)$ & $237(97.5)$ & $97(97.0)$ & & \\
\hline Yes & $9(2.6)$ & $6(2.5)$ & $3(3.0)$ & 0.078 & 0.780 \\
\hline Performance of BPs & & & & & \\
\hline No & $84(24.5)$ & $56(23.0)$ & $28(28.0)$ & & \\
\hline Yes & $259(75.5)$ & $187(77.0)$ & $72(72.0)$ & 0.941 & 0.332 \\
\hline Liver metastasis & & & & & \\
\hline No & $224(65.3)$ & $155(63.8)$ & $69(69.0)$ & 0850 & ( 357 \\
\hline Yes & $119(34.7)$ & $88(36.2)$ & $31(31.0)$ & 0.850 & 0.357 \\
\hline Brain metastasis & & & & & \\
\hline No & $307(89.5)$ & $217(89.3)$ & $90(90.0)$ & & \\
\hline Yes & $36(10.5)$ & $26(10.7)$ & $10(10.0)$ & 0.037 & 0.848 \\
\hline
\end{tabular}


TABLE 1: Continued.

\begin{tabular}{|c|c|c|c|c|c|}
\hline Subject characteristics & $\begin{array}{c}\text { Total cohort } \\
N(\%)\end{array}$ & $\begin{array}{c}\text { Construction cohort } \\
N(\%)\end{array}$ & $\begin{array}{c}\text { Validation cohort } \\
N(\%)\end{array}$ & $\chi^{2}$ & $P$ value \\
\hline \multicolumn{6}{|l|}{ Lung metastasis } \\
\hline No & $206(60.1)$ & $147(60.5)$ & $59(59.0)$ & \multirow{2}{*}{0.066} & \multirow{2}{*}{0.797} \\
\hline Yes & $137(39.9)$ & $96(39.5)$ & $41(41.0)$ & & \\
\hline \multicolumn{6}{|l|}{ Other organs metastasis } \\
\hline No & $267(77.8)$ & $191(78.6)$ & $76(76.0)$ & \multirow{2}{*}{0.278} & \multirow{2}{*}{0.598} \\
\hline Yes & $76(22.2)$ & $52(21.4)$ & $24(24.0)$ & & \\
\hline
\end{tabular}

HGB: hemoglobin; CA153: carbohydrate antigen 153; CEA: carcinoembryonic antigen; ALP: alkaline phosphatase; ER: estrogen receptor; PR: progesterone receptor; Her-2: human epidermal growth factor receptor 2; BC: breast cancer; BM: bone metastasis; SBM: synchronous bone metastasis; MBM: metachronous bone metastasis; BPs: bisphosphonates.

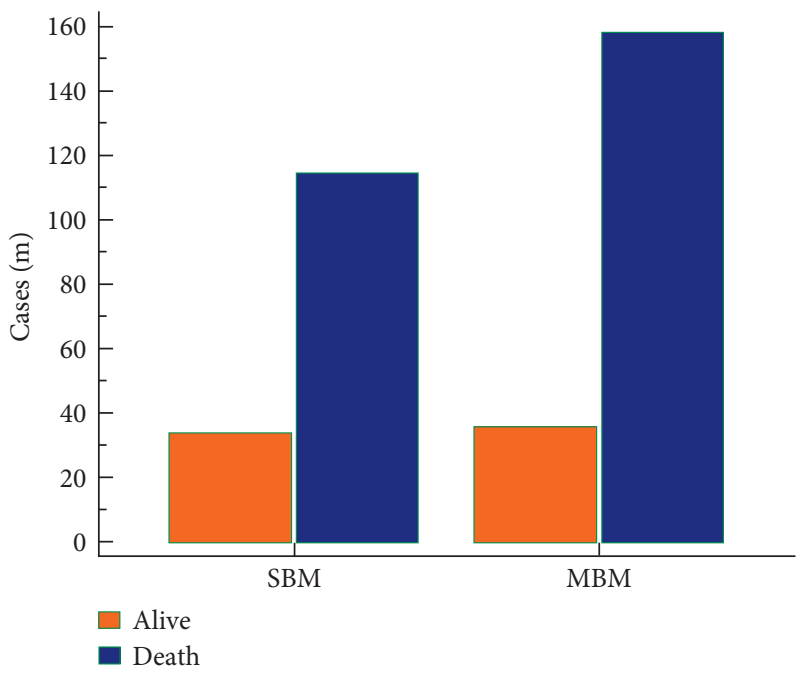

(a)

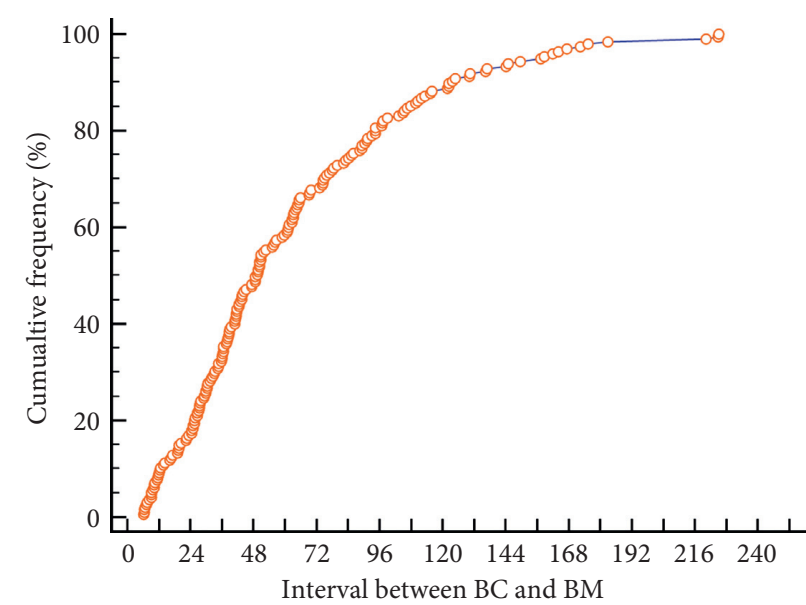

(b)

FIgURE 2: The distribution of survival outcome of synchronous bone metastasis and metachronous bone metastasis (a). The cumulative frequency of bone metastasis in metachronous bone metastasis patients (b).

lymph node metastasis, ER status, PR status, Her-2 status, Ki-67 status, surgery for BC, chemotherapy, endocrinotherapy, SBM/MBM, performance of BPs, liver metastasis, lung metastasis, and other organs metastasis. After adjusting all these characteristics in multivariate analysis, elevated ALP $(\mathrm{HR}=1.71,95 \% \mathrm{CI}: 1.16-2.51 ; P=0.006)$, no surgery for breast cancer $(\mathrm{HR}=2.19,95 \% \mathrm{CI}$ : $1.30-3.70$; $P=0.003)$, SBM (HR=1.98, 95\% CI: $1.22-3.22 ; P=0.006)$, and liver metastasis $(\mathrm{HR}=1.68,95 \% \mathrm{CI}: 1.20-2.37$; $P=0.003)$ were independent prognostic factors for worse survival. More details about the univariate and multivariate Cox regression analysis are shown in Tables 2 and 3, respectively.

3.4. Construction and Validation of Nomogram. As shown in Figure 3, the abovementioned four independent prognostic factors and other five factors (including age at diagnosis, ER status, PR status, Her-2 status, and the performance of BPs) were included to construct a prognostic nomogram. The C-index was 0.714 (95\% CI: 0.636-0.792) and the AUC of the nomogram for 1 year, 3 years, 5 years, and 10 years were $0.910,0.848,0.765$, and 0.752 , respectively (Figure 4 ). The calibration curve revealed good agreement between the predicted and observed probabilities. All the calibration curves were close to the 45-degree line (Figure 5).

In the validation cohort, the nomogram showed satisfactory strength of discrimination. The C-index was 0.705 (95\% CI: 0.705) and the AUC for 1 year, 3 years, 5 years, and 10 years were $0.831,0.766,0.689$, and 0.696 , respectively (Figure 6). Excellent ability of calibration was achieved with all calibration curves close to the 45-degree line (Figure 7).

\section{Discussion}

In the current study, a Chinese breast cancer bone metastasis cohort from the Department of Bone and Soft Tissue Tumor was described and associated prognostic factors were identified. Unlike cohorts based on the public dataset, our cohort comprised more treatment information and laboratory data. Besides, SBM and MBM were distinguished according to the time of BM. Previous studies demonstrated the impact of these variables on prognosis prediction and our study further confirmed that [19-23]. Meanwhile, these variables were incorporated to construct a predictive nomogram with satisfactory discrimination and calibration. 
TABLE 2: Univariate Cox proportional hazard regression model for analyzing associated factors in the construction cohort.

\begin{tabular}{|c|c|c|c|c|c|c|}
\hline Subject characteristics & $\mathrm{B}$ & SE & Wald & $P$ value & HR & HR 95\% Cl \\
\hline \multicolumn{7}{|l|}{ Age } \\
\hline $18-45$ & & & 11.006 & 0.004 & 1.00 & Ref \\
\hline $46-55$ & 0.169 & 0.176 & 0.916 & 0.338 & 1.18 & $0.84-1.67$ \\
\hline$>55$ & 0.590 & 0.180 & 10.675 & 0.001 & 1.80 & $1.27-2.57$ \\
\hline \multicolumn{7}{|l|}{ Marital status } \\
\hline Married & & & & & 1.00 & Ref \\
\hline Unmarried & 0.230 & 0.343 & 0.449 & 0.503 & 1.26 & $0.64-2.46$ \\
\hline \multicolumn{7}{|l|}{ History of smoking } \\
\hline Yes & & & & & 1.00 & Ref \\
\hline No & 0.172 & 0.364 & 0.224 & 0.636 & 1.19 & $0.58-2.43$ \\
\hline \multicolumn{7}{|l|}{ Alcohol consumption } \\
\hline Yes & & & & & 1.00 & Ref \\
\hline No & 0.182 & 0.342 & 0.281 & 0.596 & 1.20 & $0.61-2.35$ \\
\hline \multicolumn{7}{|l|}{ Menstrual status } \\
\hline Menstruation & & & & & 1.00 & Ref \\
\hline Menopause & -0.211 & 0.161 & 1.717 & 0.190 & 0.81 & $0.59-1.11$ \\
\hline \multicolumn{7}{|l|}{ History of abortion } \\
\hline No & & & & & 1.00 & Ref \\
\hline Yes & 0.010 & 0.157 & 0.004 & 0.952 & 1.01 & $0.74-1.38$ \\
\hline \multicolumn{7}{|l|}{ Family history of tumor } \\
\hline Yes & & & & & 1.00 & Ref \\
\hline No & -0.079 & 0.171 & 0.212 & 0.645 & 0.92 & $0.66-1.29$ \\
\hline \multicolumn{7}{|l|}{ ABO blood type } \\
\hline A type & & & 6.885 & 0.076 & 1.00 & Ref \\
\hline B type & 0.092 & 0.188 & 0.240 & 0.624 & 1.10 & $0.76-1.59$ \\
\hline AB type & 0.558 & 0.235 & 5.656 & 0.017 & 1.75 & $1.10-2.77$ \\
\hline O type & -0.002 & 0.204 & 0 & 0.994 & 1.00 & $0.67-1.49$ \\
\hline \multicolumn{7}{|l|}{ HGB } \\
\hline $115-150 \mathrm{~g} / \mathrm{L}$ & & & 2.906 & 0.234 & 1.00 & Ref \\
\hline$<115 \mathrm{~g} / \mathrm{L}$ & 0.098 & 0.193 & 0.258 & 0.611 & 1.10 & $0.76-1.61$ \\
\hline$>150 \mathrm{~g} / \mathrm{L}$ & 0.758 & 0.457 & 2.758 & 0.097 & 2.14 & $0.87-5.22$ \\
\hline \multicolumn{7}{|l|}{ CA153 } \\
\hline $0-25 \mathrm{U} / \mathrm{ml}$ & & & & & 1.00 & Ref \\
\hline$>25 \mathrm{U} / \mathrm{ml}$ & -0.342 & 0.158 & 4.684 & 0.030 & 0.71 & $0.52-0.97$ \\
\hline \multicolumn{7}{|l|}{ CEA } \\
\hline $0-5 \mathrm{ng} / \mathrm{ml}$ & & & & & 1.00 & Ref \\
\hline$>5 \mathrm{ng} / \mathrm{ml}$ & -0.142 & 0.146 & 0.946 & 0.331 & 0.87 & $0.65-1.16$ \\
\hline \multicolumn{7}{|l|}{$\overline{A L P}$} \\
\hline $50-135 \mathrm{U} / \mathrm{L}$ & & & 7.375 & 0.025 & 1.00 & Ref \\
\hline$<50 \mathrm{U} / \mathrm{L}$ & 0.118 & 0.267 & 0.197 & 0.657 & 1.13 & $0.67-1.90$ \\
\hline$>135 \mathrm{U} / \mathrm{L}$ & 0.451 & 0.166 & 7.373 & 0.007 & 1.57 & $1.13-2.18$ \\
\hline \multicolumn{7}{|l|}{ Serum calcium } \\
\hline $2.10-2.55 \mathrm{mmol} / \mathrm{L}$ & & & 1.613 & 0.446 & 1.00 & Ref \\
\hline$<2.10 \mathrm{mmol} / \mathrm{L}$ & 0.185 & 0.232 & 0.636 & 0.425 & 1.20 & $0.76-1.89$ \\
\hline$>2.55 \mathrm{mmol} / \mathrm{L}$ & 0.207 & 0.185 & 1.251 & 0.263 & 1.23 & $0.86-1.77$ \\
\hline \multicolumn{7}{|l|}{ Pathology } \\
\hline Ductal carcinoma & & & & & 1.00 & Ref \\
\hline Others & 0.227 & 0.152 & 2.240 & 0.134 & 1.26 & $0.93-1.69$ \\
\hline \multicolumn{7}{|l|}{ Grade } \\
\hline I-II & & & & & 1.00 & Ref \\
\hline III & 0.470 & 0.160 & 8.654 & 0.003 & 1.60 & $1.17-2.19$ \\
\hline \multicolumn{7}{|l|}{ Tumor size } \\
\hline$<2 \mathrm{~cm}$ & & & 0.816 & 0.665 & 1.00 & Ref \\
\hline $2-5 \mathrm{~cm}$ & 0.073 & 0.165 & 0.196 & 0.658 & 1.08 & $0.78-1.49$ \\
\hline$>5 \mathrm{~cm}$ & 0.214 & 0.238 & 0.814 & 0.367 & 1.24 & $0.78-1.97$ \\
\hline Lymph node metastasis & & & & & & \\
\hline Yes & & & & & 1.00 & Ref \\
\hline No & -0.459 & 0.191 & 5.766 & 0.016 & 0.63 & $0.44-0.92$ \\
\hline
\end{tabular}


TABle 2: Continued.

\begin{tabular}{|c|c|c|c|c|c|c|}
\hline Subject characteristics & $\mathrm{B}$ & SE & Wald & $P$ value & HR & HR 95\% CI \\
\hline \multicolumn{7}{|l|}{ ER status } \\
\hline Positive & & & & & 1.00 & Ref \\
\hline Negative & 0.330 & 0.154 & 4.615 & 0.032 & 1.39 & $1.03-1.88$ \\
\hline \multicolumn{7}{|l|}{ PR status } \\
\hline Positive & & & & & 1.00 & Ref \\
\hline Negative & 0.396 & 0.146 & 7.390 & 0.007 & 1.49 & $1.12-1.98$ \\
\hline \multicolumn{7}{|l|}{ Her-2 status } \\
\hline Positive & & & & & 1.00 & Ref \\
\hline Negative & -0.411 & 0.172 & 5.735 & 0.017 & 0.66 & $0.47-0.93$ \\
\hline \multicolumn{7}{|l|}{ Ki-67 status } \\
\hline Positive & & & & & 1.00 & Ref \\
\hline Negative & 0.522 & 0.176 & 8.791 & 0.003 & 1.69 & $1.19-2.38$ \\
\hline \multicolumn{7}{|l|}{ Surgery for $\mathrm{BC}$} \\
\hline Yes & & & & & 1.00 & Ref \\
\hline No & 1.372 & 0.181 & 57.553 & $<0.001$ & 3.94 & $2.77-5.62$ \\
\hline \multicolumn{7}{|l|}{ Chemotherapy } \\
\hline No & & & & & 1.00 & Ref \\
\hline Yes & -0.802 & 0.344 & 5.430 & 0.020 & 0.45 & $0.23-0.88$ \\
\hline \multicolumn{7}{|l|}{ Radiotherapy } \\
\hline No & & & & & 1.00 & Ref \\
\hline Yes & -0.036 & 0.150 & 0.058 & 0.810 & 0.97 & $0.72-1.30$ \\
\hline \multicolumn{7}{|l|}{ Endocrinotherapy } \\
\hline No & & & & & 1.00 & Ref \\
\hline Yes & -0.473 & 0.147 & 10.35 & 0.001 & 0.62 & $0.47-0.83$ \\
\hline \multicolumn{7}{|l|}{ Targeted therapy } \\
\hline No & & & & & 1.00 & Ref \\
\hline Yes & 0.211 & 0.211 & 0.998 & 0.318 & 1.24 & $0.82-1.87$ \\
\hline \multicolumn{7}{|l|}{ Time of BM } \\
\hline SBM & & & & & 1.00 & Ref \\
\hline MBM & -1.104 & 0.163 & 45.613 & $<0.001$ & 0.33 & $0.24-0.46$ \\
\hline \multicolumn{7}{|l|}{ Radiotherapy for BM } \\
\hline No & & & & & 1.00 & Ref \\
\hline Yes & 0.085 & 0.186 & 0.208 & 0.649 & 1.09 & $0.76-1.57$ \\
\hline \multicolumn{7}{|l|}{ Pathological fracture } \\
\hline No & & & & & 1.00 & Ref \\
\hline Yes & 0.300 & 0.263 & 1.304 & 0.254 & 1.35 & $0.81-2.26$ \\
\hline \multicolumn{7}{|l|}{ Spinal cord compression } \\
\hline No & & & & & 1.00 & Ref \\
\hline Yes & -0.066 & 0.416 & 0.026 & 0.873 & 0.94 & $0.41-2.11$ \\
\hline \multicolumn{7}{|l|}{ Surgery for BM } \\
\hline No & & & & & 1.00 & Ref \\
\hline Yes & 0.077 & 0.455 & 0.029 & 0.865 & 1.08 & $0.44-2.63$ \\
\hline \multicolumn{7}{|l|}{ Performance of BPs } \\
\hline No & & & & & 1.00 & \\
\hline Yes & -0.382 & 0.166 & 5.288 & 0.021 & 0.68 & $0.49-0.95$ \\
\hline \multicolumn{7}{|l|}{ Liver metastasis } \\
\hline No & & & & & 1.00 & Ref \\
\hline Yes & 0.469 & 0.147 & 10.140 & 0.001 & 1.60 & $1.2-2.13$ \\
\hline \multicolumn{7}{|l|}{ Brain metastasis } \\
\hline No & & & & & 1.00 & Ref \\
\hline Yes & 0.355 & 0.212 & 2.807 & 0.094 & 1.43 & $0.94-2.16$ \\
\hline Lung metastasis & & & & & & \\
\hline No & & & & & 1.00 & Ref \\
\hline Yes & -0.311 & 0.150 & 4.335 & 0.037 & 0.73 & $0.55-0.98$ \\
\hline Other organs metastasi & & & & & & \\
\hline No & & & & & 1.00 & Ref \\
\hline Yes & -0.366 & 0.179 & 4.182 & 0.041 & 0.69 & $0.49-0.99$ \\
\hline
\end{tabular}

HGB: hemoglobin; CA153: carbohydrate antigen 153; CEA: carcinoembryonic antigen; ALP: alkaline phosphatase; ER: estrogen receptor; PR: progesterone receptor; Her-2: human epidermal growth factor receptor 2; BC: breast cancer; BM: bone metastasis; SBM: synchronous bone metastasis; MBM: metachronous bone metastasis; BPs: bisphosphonates. 
TABLE 3: Multivariate Cox proportional hazard regression model for analyzing the independent prognostic factors in the construction cohort.

\begin{tabular}{|c|c|c|c|c|c|c|}
\hline Subject characteristics & $\mathrm{B}$ & SE & Wald & $P$ value & HR & HR 95\% CI \\
\hline \multicolumn{7}{|l|}{ Age } \\
\hline $18-45$ & & & 0.332 & 0.847 & 1.00 & Ref \\
\hline $46-55$ & 0.087 & 0.192 & 0.206 & 0.650 & 1.09 & $0.75-1.59$ \\
\hline$>55$ & -0.023 & 0.224 & 0.010 & 0.918 & 0.98 & $0.63-1.52$ \\
\hline \multicolumn{7}{|l|}{ ABO blood type } \\
\hline A type & & & 5.191 & 0.158 & 1.00 & Ref \\
\hline B type & 0.315 & 0.204 & 2.376 & 0.123 & 1.37 & $0.92-2.04$ \\
\hline AB type & 0.495 & 0.265 & 3.503 & 0.061 & 1.64 & $0.98-2.76$ \\
\hline O type & 0.047 & 0.231 & 0.042 & 0.837 & 1.05 & $0.67-1.65$ \\
\hline \multicolumn{7}{|l|}{ CA153 } \\
\hline $0-25 \mathrm{U} / \mathrm{ml}$ & & & & & 1.00 & Ref \\
\hline$>25 \mathrm{U} / \mathrm{ml}$ & -0.268 & 0.189 & 2.005 & 0.157 & 0.77 & $0.53-1.11$ \\
\hline \multicolumn{7}{|l|}{ ALP } \\
\hline $50-135 \mathrm{U} / \mathrm{L}$ & & & 7.448 & 0.024 & 1.00 & Ref \\
\hline$<50 \mathrm{U} / \mathrm{L}$ & 0.090 & 0.290 & 0.097 & 0.756 & 1.09 & $0.62-1.93$ \\
\hline$>135 \mathrm{U} / \mathrm{L}$ & 0.536 & 0.197 & 7.448 & 0.006 & 1.71 & $1.16-2.51$ \\
\hline \multicolumn{7}{|l|}{ Grade } \\
\hline I-II & & & & & 1.00 & Ref \\
\hline III & 0.124 & 0.197 & 0.392 & 0.531 & 1.13 & $0.77-1.67$ \\
\hline \multicolumn{7}{|l|}{ Lymph node metastasis } \\
\hline Yes & & & & & 1.00 & Ref \\
\hline No & -0.075 & 0.209 & 0.129 & 0.719 & 0.93 & $0.62-1.40$ \\
\hline \multicolumn{7}{|l|}{ ER status } \\
\hline Positive & & & & & 1.00 & Ref \\
\hline Negative & -0.066 & 0.214 & 0.094 & 0.759 & 0.94 & $0.62-1.42$ \\
\hline \multicolumn{7}{|l|}{ PR status } \\
\hline Positive & & & & & 1.00 & Ref \\
\hline Negative & 0.289 & 0.191 & 2.303 & 0.129 & 1.34 & $0.92-1.94$ \\
\hline \multicolumn{7}{|l|}{ Her-2 status } \\
\hline Positive & & & & & 1.00 & Ref \\
\hline Negative & -0.207 & 0.197 & 1.106 & 0.293 & 0.81 & $0.55-1.20$ \\
\hline \multicolumn{7}{|l|}{ Ki-67 status } \\
\hline Positive & & & & & 1.00 & Ref \\
\hline Negative & 0.341 & 0.207 & 2.714 & 0.099 & 1.41 & $0.94-2.11$ \\
\hline \multicolumn{7}{|l|}{ Surgery for $\mathrm{BC}$} \\
\hline Yes & & & & & 1.00 & Ref \\
\hline No & 0.786 & 0.267 & 8.655 & 0.003 & 2.19 & $1.30-3.70$ \\
\hline \multicolumn{7}{|l|}{ Chemotherapy } \\
\hline No & & & & & 1.00 & \\
\hline Yes & -0.373 & 0.406 & 0.845 & 0.358 & 0.69 & $0.31-1.53$ \\
\hline \multicolumn{7}{|l|}{ Endocrinotherapy } \\
\hline No & & & & & 1.00 & Ref \\
\hline Yes & -0.080 & 0.190 & 0.178 & 0.673 & 0.92 & $0.64-1.34$ \\
\hline \multicolumn{7}{|l|}{ Time of BM } \\
\hline MBM & & & & & 1.00 & Ref \\
\hline SBM & 0.684 & 0.247 & 7.646 & 0.006 & 1.98 & $1.22-3.22$ \\
\hline \multicolumn{7}{|l|}{ Performance of BPs } \\
\hline No & & & & & 1.00 & Ref \\
\hline Yes & -0.308 & 0.200 & 2.364 & 0.124 & 0.74 & $0.50-1.09$ \\
\hline \multicolumn{7}{|l|}{ Liver metastasis } \\
\hline No & & & & & 1.00 & Ref \\
\hline Yes & 0.521 & 0.175 & 8.872 & 0.003 & 1.68 & $1.20-2.37$ \\
\hline \multicolumn{7}{|l|}{ Lung metastasis } \\
\hline No & & & & & 1.00 & Ref \\
\hline Yes & -0.216 & 0.165 & 1.725 & 0.189 & 0.81 & $0.58-1.11$ \\
\hline
\end{tabular}


TABLE 3: Continued.

\begin{tabular}{lcccccc}
\hline Subject characteristics & B & SE & Wald & $P$ value & HR & HR 95\% CI \\
\hline $\begin{array}{l}\text { Other } \text { organs metastasis } \\
\text { No }\end{array}$ & & & & & 1.00 & Ref \\
$\quad$ Yes & -0.288 & 0.197 & 2.139 & 0.144 & 0.75 & $0.51-1.10$ \\
\hline
\end{tabular}

CA153: carbohydrate antigen 153; ALP: alkaline phosphatase; ER: estrogen receptor; PR: progesterone receptor; Her-2: human epidermal growth factor receptor 2; BC: breast cancer; BM: bone metastasis; SBM: synchronous bone metastasis; MBM: metachronous bone metastasis; BPs: bisphosphonates.

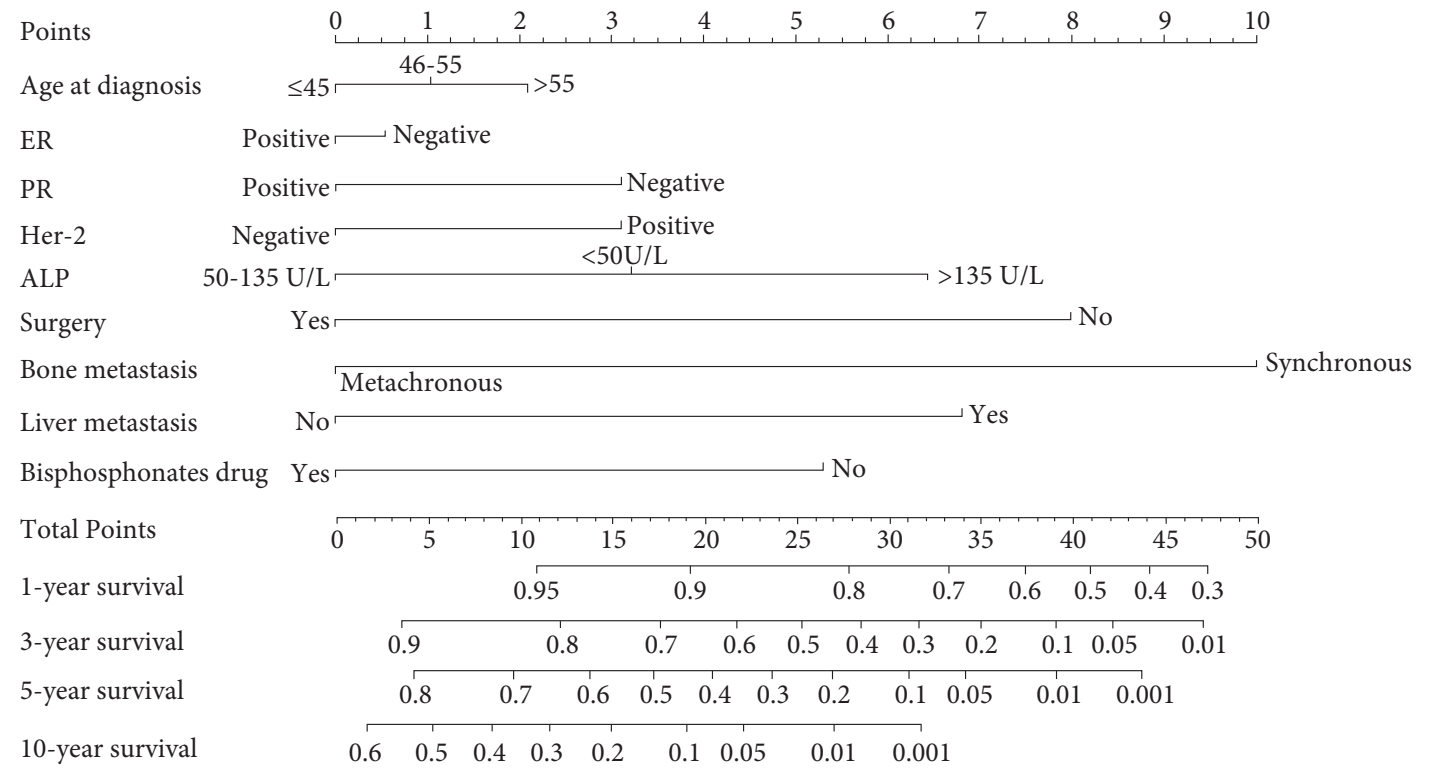

FIgURE 3: The nomogram to predict survival outcomes for breast cancer patients with bone metastasis.

As one of the biochemical bone markers, ALP indicated tumor burden and distant metastasis [24]. Consistent with previous studies, elevated ALP was an independent prognostic factor in the present study [23, 25]. Patients with elevated ALP were at 1.71-fold risk of death compared with those with normal ALP. In a retrospective study focusing on bone-only metastasis patients, the median OS were 31.0 and 15.0 months for patients with normal and elevated serum ALP $(P=0.002)$ [22]. Better median OS (48.0 months) was suggested among patients with elevated ALP in the present study, which may be attributed to the high proportion of MBM patients.

In recent years, the definition of BM (SBM/MBM) was a global concern in distant metastasis [26, 27]. Sufficient evidence showed the significant difference between SBM and MBM in clinicopathologic characteristics and survival outcome $[21,26,28]$. However, SBM and MBM were still not well defined, which might be the main reason for inconsistent conclusions in previous studies [26, 27]. Although five characteristics (including age at diagnosis, ER status, PR status, Her-2 status, and the performance of BPs) were not independent prognostic factors in the present study, they were integrated in the nomogram after considering their significant effects on prognosis in literatures $[6,29,30]$.
Several nomograms were constructed based on SEER database to predict the prognosis of $\mathrm{MBC}$ patients at diagnosis [18, 19]. A retrospective study comprising 5,860 BCBM patients reported prognostic nomograms to predict overall survival (OS) and cancer-specific survival (CSS) [19]. The C-index of OS and CSS in the construction cohort was 0.705 and 0.710 , respectively. Calibration plots showed the prediction curves were close to 45 degrees in two models [19]. Another SEER-based study enrolled 7,199 stage IV BC patients to predict 1-year and 3-year OS rates, which can stratify patients into different risk groups for clinic demands [18]. These two nomograms were well established and presented good discrimination and calibration. However, due to the limitation of SEER database, the patients with MBM were not involved. Thus, the models cannot be widely used in clinic. At the same time, seldom Chinese patients were recorded; considering racial disparity in cancer, a cohort based on Chinese population is needed.

The present study had some limitations. First, as with many retrospective studies, there was inherent selection bias that cannot be assessed or avoided. Second, missing data were completed by statistical methods, which may lead to the reduction of sample's representativeness. Last but not least, limited Department of Bone and Soft Tissue Tumor was established in China; only internal validation was 

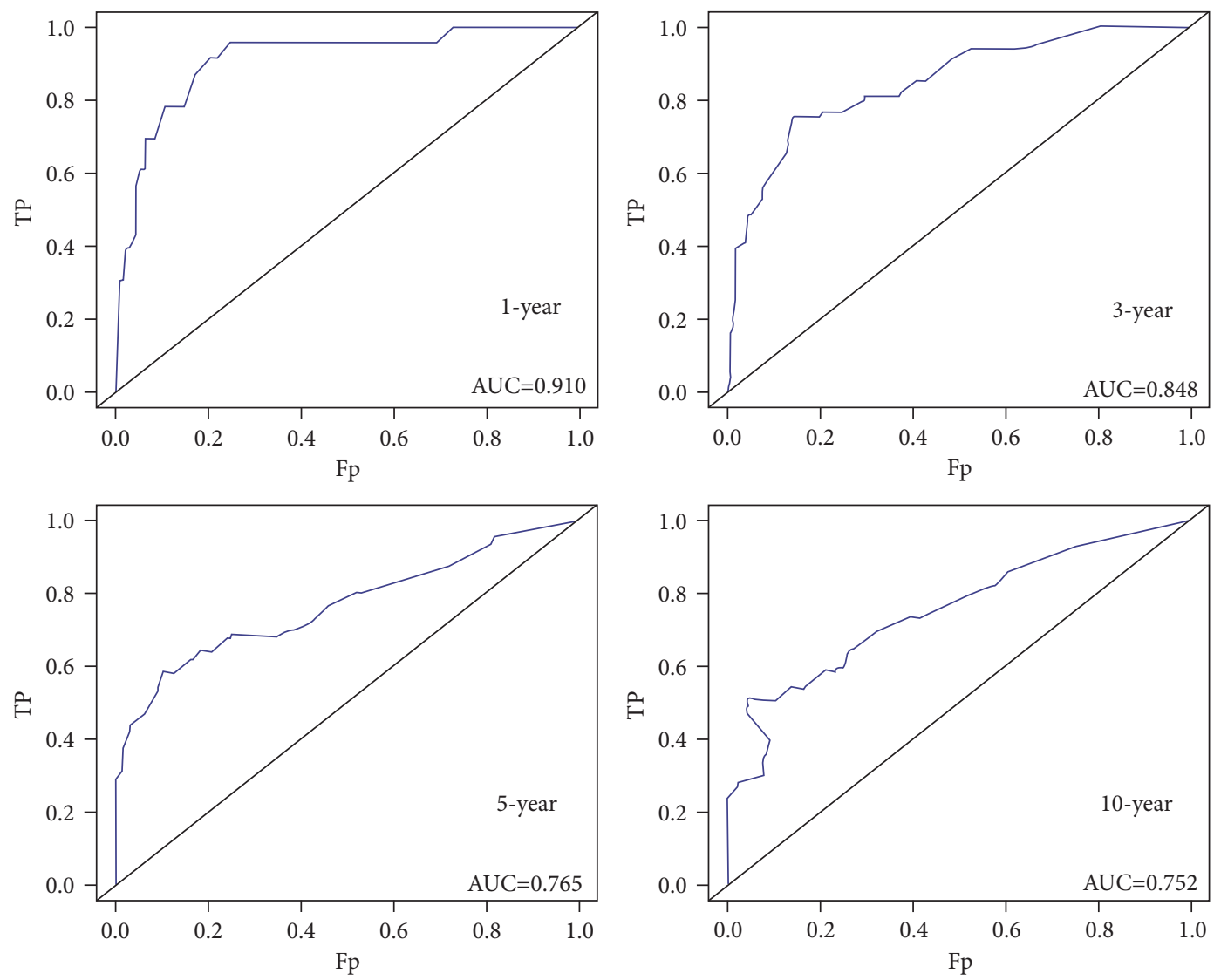

FIgURE 4: The ROC curves to predict 1-year, 3-year, 5-year, and 10-year survival in construction cohort.

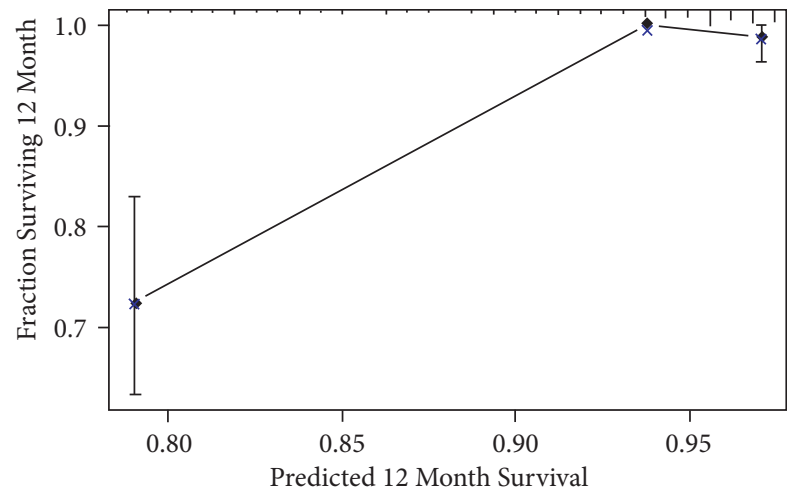

$\mathrm{n}=243 \mathrm{~d}=190 \mathrm{p}=9,80$ subjects per group $\mathrm{X}$ - resampling optimism added, $\mathrm{B}=1000$ Gray: ideal Based on observed-predicted

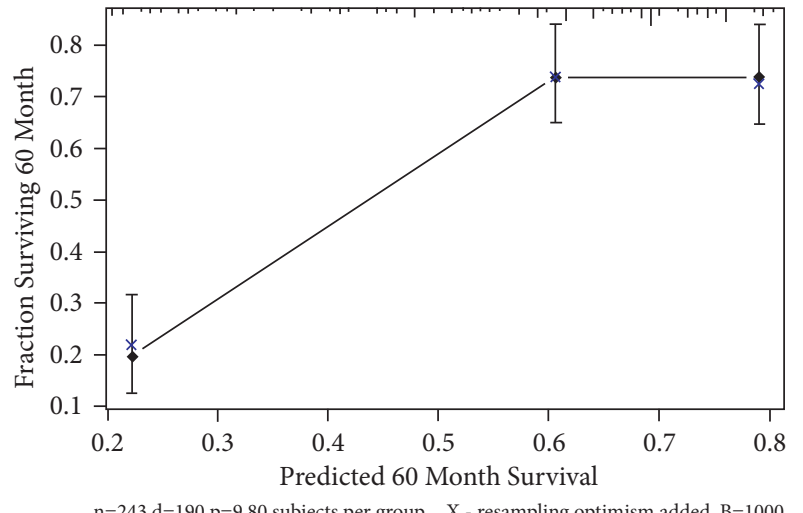

$\mathrm{n}=243 \mathrm{~d}=190 \mathrm{p}=9,80$ subjects per group $\mathrm{X}$ - resampling optimism added, $\mathrm{B}=1000$ Gray: ideal

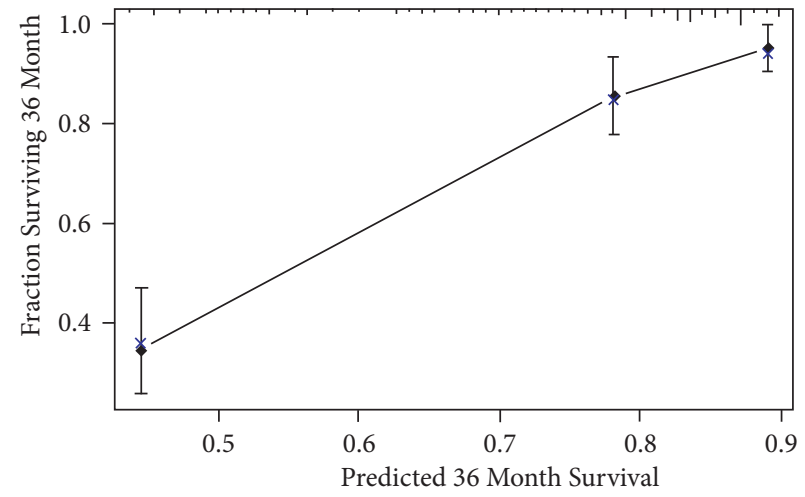

$\mathrm{n}=243 \mathrm{~d}=190 \mathrm{p}=9,80$ subjects per group $\mathrm{X}$ - resampling optimism added, $\mathrm{B}=1000$ Gray: ideal Based on observed-predicted

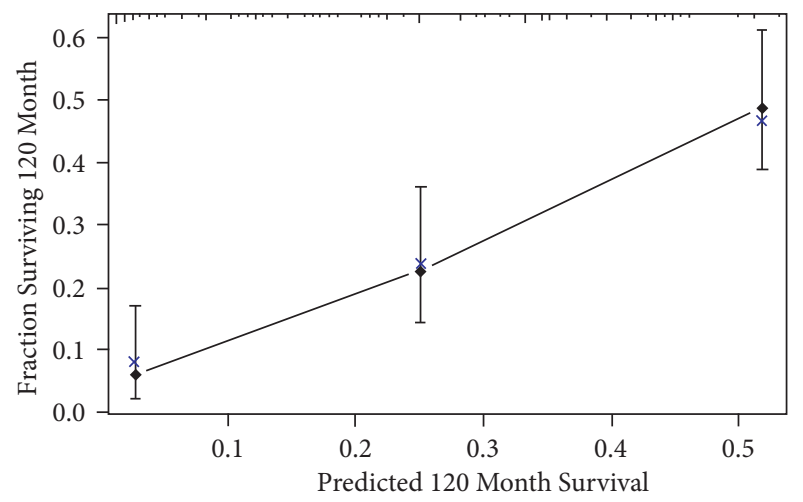

$\mathrm{n}=243 \mathrm{~d}=190 \mathrm{p}=9,80$ subjects per group $\quad \mathrm{X}$ - resampling optimism added, $\mathrm{B}=1000$ Gray: ideal

Based on observed-predicted

Figure 5: The calibration curves in construction cohort. 

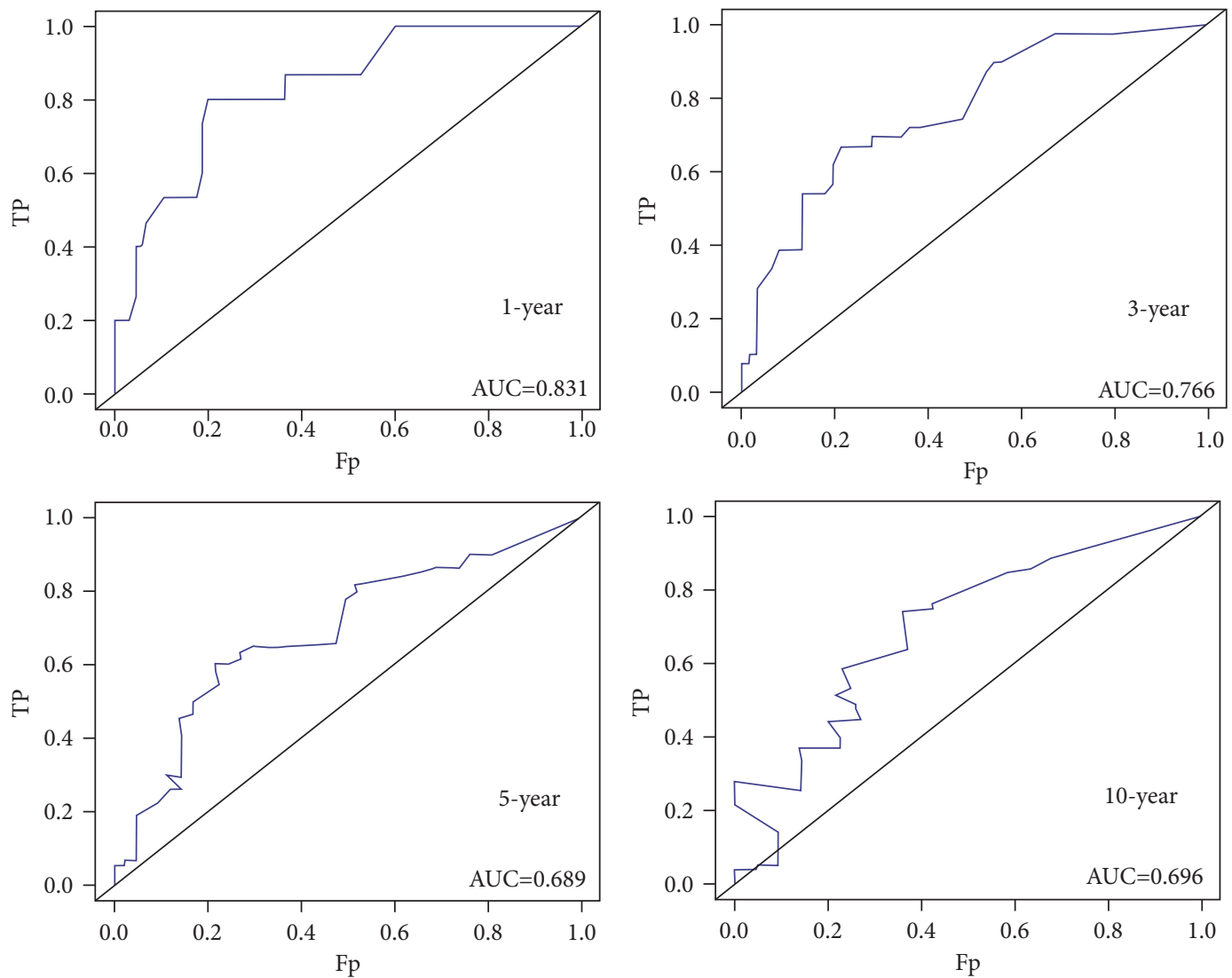

FIgURE 6: The ROC curves to predict 1-year, 3-year, 5-year, and 10-year survival in validation cohort.
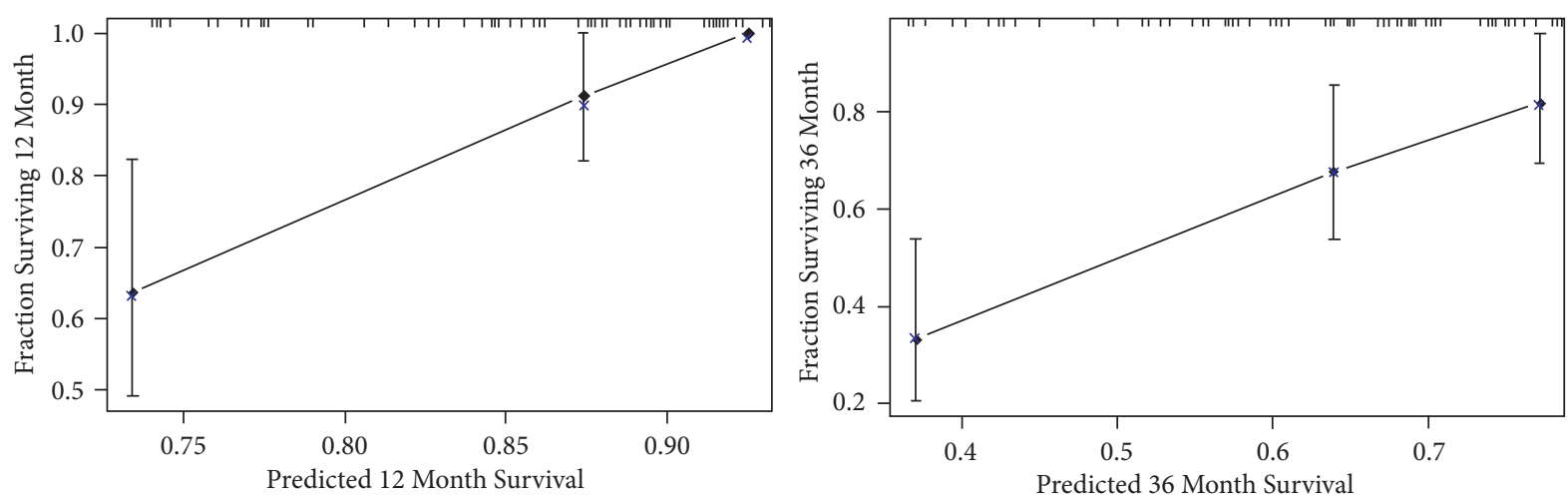

$n=100 d=83 p=1,30$ subjects per group $\quad X$ - resampling optimism added, $B=1000$ Based on observed-predicted $n=100 d=83 p=1,30$ subjects per group $\quad X-$ resampling optimism added, $B=1000$ Gray: ideal Based on observed-predicted

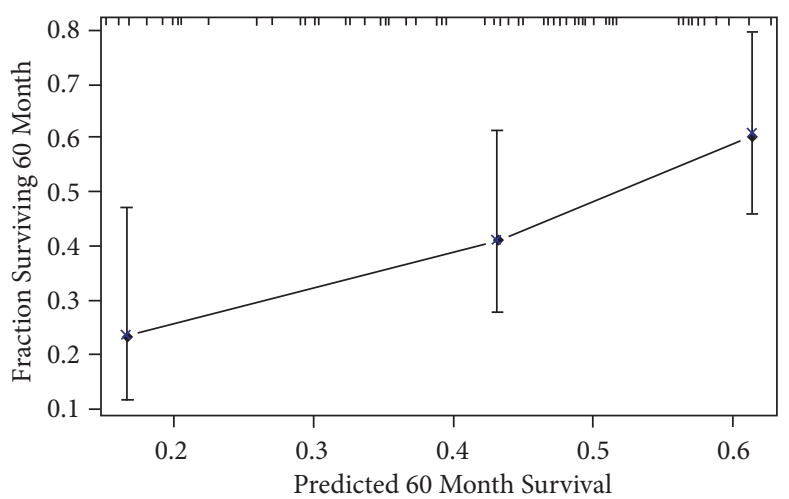

$n=100 \mathrm{~d}=83 \mathrm{p}=1,30$ subjects per group $\quad \mathrm{X}$ - resampling optimism added, $\mathrm{B}=1000$ Gray: ideal Based on observed-predicted

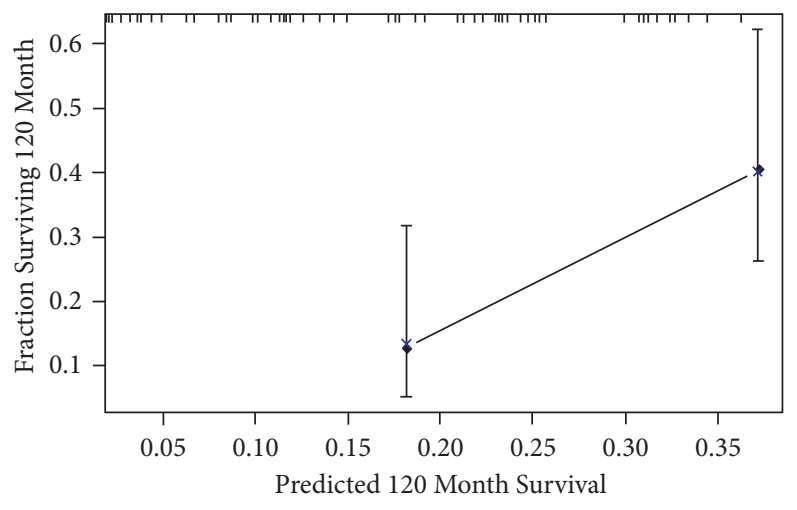

$\mathrm{n}=100 \mathrm{~d}=83 \mathrm{p}=1,30$ subjects per group $\quad \mathrm{X}$ - resampling optimism added, $\mathrm{B}=1000$ Gray: ideal $\quad$ Based on observed-predicted

FIgURE 7: The calibration curves in validation cohort. 
performed. The generalizability of model should be validated in further studies.

\section{Conclusions}

The current study provided a perspective on prognosis of BCBM. Elevated ALP, no surgery for breast cancer, synchronous bone metastasis, and liver metastasis were four independent prognostic factors for poor survival. A predictive nomogram was constructed and validated. The validation showed good performance. In the Department of Surgery, for the BM patients with potential surgical indication, these findings can be potentially used in survival estimation and individualized treatment planning generation.

\section{Data Availability}

The datasets generated during and/or analysed during the current study are available from the corresponding author on reasonable request.

\section{Conflicts of Interest}

The authors declare that there are no conflicts of interest regarding the publication of this article.

\section{Authors' Contributions}

Yao $\mathrm{Xu}$ and Haixiao $\mathrm{Wu}$ contributed equally to this work.

\section{Acknowledgments}

The present study was sponsored by the Natural Science Foundation of China (81801781, 82072004, 81903398, and $82011530050)$.

\section{References}

[1] R. L. Siegel, K. D. Miller, H. E. Fuchs, and A. Jemal, "Cancer statistics, 2021," CA: A Cancer Journal for Clinicians, vol. 71, no. 1, pp. 7-33, 2021.

[2] N. Pashayan, A. C. Antoniou, U. Ivanus et al., "Personalized early detection and prevention of breast cancer: ENVISION consensus statement," Nature Reviews Clinical Oncology, vol. 17, no. 11, pp. 687-705, 2020.

[3] J. S. Cardoso, W. Silva, and M. J. Cardoso, "Evolution, current challenges, and future possibilities in the objective assessment of aesthetic outcome of breast cancer locoregional treatment," The Breast, vol. 49, pp. 123-130, 2020.

[4] J. A. Malmgren, G. S. Calip, M. K. Atwood, M. Mayer, and H. G. Kaplan, "Metastatic breast cancer survival improvement restricted by regional disparity: surveillance, epidemiology, and end results and institutional analysis: 1990 to 2011," Cancer, vol. 126, no. 2, pp. 390-399, 2020.

[5] N. Niikura, J. Liu, N. Hayashi et al., "Treatment outcome and prognostic factors for patients with bone-only metastases of breast cancer: a single-institution retrospective analysis," The Oncologist, vol. 16, no. 2, pp. 155-164, 2011.

[6] S. G. Ahn, H. M. Lee, S.-H. Cho et al., "Prognostic factors for patients with bone-only metastasis in breast cancer," Yonsei Medical Journal, vol. 54, no. 5, p. 1168, 2013.
[7] R. P. Piggott, P. S. Waters, and M. J. Kerin, "The influence of breast cancer subtype on bone metastases development and survival in women with metastatic breast cancer," Irish Journal of Medical Science, vol. 186, pp. 97-102, 2017.

[8] X. Li, X. Zhang, J. Liu, and Y. Shen, "Prognostic factors and survival according to tumour subtype in women presenting with breast cancer bone metastases at initial diagnosis: a SEER-based study," BMC Cancer, vol. 20, 2020.

[9] C. Zhao, Z. Zhang, N. Zhong et al., "Outcomes and prognostic factors for surgically treated patients with breast cancer spine metastases," Journal of Bone Oncology, vol. 12, pp. 38-43, 2018.

[10] A. Parkes, C. L. Warneke, K. Clifton et al., "Prognostic factors in patients with metastatic breast cancer with bone-only metastases," The Oncologist, vol. 23, no. 11, pp. 1282-1288, 2018.

[11] K. Cetin, C. F. Christiansen, C. Sværke, J. B. Jacobsen, and H. T. Sørensen, "Survival in patients with breast cancer with bone metastasis: a Danish population-based cohort study on the prognostic impact of initial stage of disease at breast cancer diagnosis and length of the bone metastasis-free interval," BMJ Open, vol. 5, Article ID e007702, 2015.

[12] R. Foerster, T. Bruckner, T. Bostel, I. Schlampp, J. Debus, and H. Rief, "Prognostic factors for survival of women with unstable spinal bone metastases from breast cancer," Radiation Oncology, vol. 10, no. 1, p. 144, 2015.

[13] Y.-H. Lee, H. Bang, and D. J. Kim, "How to establish clinical prediction models," Endocrinology and Metabolism, vol. 31, no. 1 , pp. 38-44, 2016.

[14] G. S. Collins, J. B. Reitsma, D. G. Altman, and K. G. M. Moons, "Transparent reporting of a multivariable prediction model for individual prognosis or diagnosis (TRIPOD): the TRIPOD statement," BMJ, vol. 350, p. g7594, 2015.

[15] C. Zhang, M. Mao, X. Guo et al., "Nomogram based on homogeneous and heterogeneous associated factors for predicting bone metastases in patients with different histological types of lung cancer," BMC Cancer, vol. 19, no. 1, p. 238, 2019.

[16] X. Wang, M. Mao, G. Xu et al., "The incidence, associated factors, and predictive nomogram for early death in stage IV colorectal cancer," International Journal of Colorectal Disease, vol. 34, no. 7, pp. 1189-1201, 2019.

[17] Y. Xu, G. Xu, H. Wu et al., "The nomogram for early death in patients with bone and soft tissue tumors," Journal of Cancer, vol. 11, no. 18, pp. 5359-5370, 2020.

[18] S. Li, J. Zhao, L. Zhu, F. Su, and K. Chen, "Development and validation of a nomogram predicting the overall survival of stage IV breast cancer patients," Cancer Medicine, vol. 6, no. 11, pp. 2586-2594, 2017.

[19] Z. Wang, Y. Cheng, S. Chen et al., "Novel prognostic nomograms for female patients with breast cancer and bone metastasis at presentation," Annals of Translational Medicine, vol. 8, no. 5, p. 197, 2020.

[20] S. Turanli, "The role of surgery in breast cancer patients with isolated bone metastases at the time of diagnosis," Journal of Surgical Oncology, vol. 100, no. 2, pp. 95-99, 2009.

[21] S. Turanli, "Importance of the development time of isolated bone metastasis in breast cancer," Langenbeck's Archives of Surgery, vol. 397, no. 6, pp. 967-972, 2012.

[22] C. Nieder, A. Dalhaug, A. Pawinski, B. Mannsåker, and E. Haukland, "Survival after palliative radiotherapy in patients with breast cancer and bone-only metastases," In Vivo, vol. 30, no. 6, pp. 879-884, 2016. 
[23] B. Chen, D. Dai, H. Tang et al., "Pre-treatment serum alkaline phosphatase and lactate dehydrogenase as prognostic factors in triple negative breast cancer," Journal of Cancer, vol. 7, no. 15 , pp. 2309-2316, 2016

[24] W. X. Du, S. F. Duan, J. J. Chen, J. F. Huang, L. M. Yin, and P. J. Tong, "Serum bone-specific alkaline phosphatase as a biomarker for osseous metastases in patients with malignant carcinomas: a systematic review and meta-analysis," Journal of Cancer Research and Therapeutics, vol. 10, pp. C140-C143, 2014.

[25] P. S. Blanchette, D. N. Desautels, G. R. Pond et al., "Factors influencing survival among patients with HER2-positive metastatic breast cancer treated with trastuzumab," Breast Cancer Research and Treatment, vol. 170, no. 1, pp. 169-177, 2018.

[26] C.-X. Ma, X. Guan, R. Wei et al., "The distinction of clinicopathological characteristics, treatment strategy and outcome in colorectal cancer patients with synchronous vs. metachronous bone metastasis," Frontiers in Oncology, vol. 10, p. 974, 2020.

[27] J. Mikami, Y. Kimura, Y. Makari et al., "Clinical outcomes and prognostic factors for gastric cancer patients with bone metastasis," World Journal of Surgical Oncology, vol. 15, no. 1, p. 8, 2017.

[28] W. Ma, X. Wang, G. Xu et al., "Distant metastasis prediction via a multi-feature fusion model in breast cancer," Aging, vol. 12, no. 18, pp. 18151-18162, 2020.

[29] A. Purushotham, E. Shamil, M. Cariati et al., "Age at diagnosis and distant metastasis in breast cancer-a surprising inverse relationship," European Journal of Cancer, vol. 50, no. 10, pp. 1697-1705, 2014.

[30] A. Yazdani, S. Dorri, A. Atashi, H. Shirafkan, and H. Zabolinezhad, "Bone metastasis prognostic factors in breast cancer," Breast Cancer: Basic and Clinical Research, vol. 13, Article ID 1178223419830978, 2019. 